\title{
4H-SiC PIN-type Semiconductor Detector for Fast Neutron Detection
}

\author{
Jang Ho HA ${ }^{1 *}$, Sang Mook KANG ${ }^{1}$, Han Soo KIM ${ }^{1}$, Se-Hwan PARK ${ }^{1}$, Nam Ho LEE ${ }^{1}$, Tae-Yong SONG ${ }^{1}$, Jae Hyung LEE ${ }^{1}$ \\ Hyeonseo PARK ${ }^{2}$ and Jungho KIM ${ }^{2}$ \\ ${ }^{1}$ Korea Atomic Energy Research Institute, 1045 Daedeokdaero, Yusunggu, Daejeon, 305-353, Korea \\ ${ }^{2}$ Korea Research Institute of Standards and Science, 209, Gajungro, Yusunggu, Daejon, 305-340, Korea
}

\begin{abstract}
Radiation detectors based on semiconductors like Silicon Carbide ( $\mathrm{SiC}$ ), Aluminum Nitride (AlN), Boron Nitride $(\mathrm{BN})$ and Gallium Arsenide (GaAs) with a large energy band gap are the most promising ionizing radiation detectors for high temperature and in harsh radiation fields. The present work focused on the development of a radiation resistive neutron semiconductor detector based on a wide band-gap 4H-SiC semiconductor, operating at a zero biased voltage by using a strong internal electric field. The self-biased detector structure was a PIN device, which was fabricated in KAERI based on the wafer provided by Cree Co.. The device was structured as 4 layers, which consisted of $0.5 \mu \mathrm{m}$ of P-layer, $2 \mu \mathrm{m}$ of I-layer, $30 \mu \mathrm{m}$ of N-layer on N-type substrate with $350 \mu \mathrm{m}$ in thickness. Neutron detection responses were measured up to a $1.1 \times 10^{6} \mathrm{n} / \mathrm{sec}-\mathrm{cm}^{2}$ by using an Am-Be neutron source at a self-powered operation. The absolute neutron detection efficiency was determined as $5.1 \times 10^{-4}$ at a $5.0 \mathrm{MeV}$ in neutron energy.
\end{abstract}

KEYWORDS: Solid State Detector, Silicon Carbide (SiC), fast neutron response, Self biased operation

\section{Introduction}

The harsh radiation environments such as a nuclear reactor core, high energy physics experiments, or outer space need detectors with radiation hard properties. Fast neutron detectors based on wide band-gap semiconductors are being developed for quantification of fissionable materials. ${ }^{1-3)}$ Neutron flux measurement for in-core of nuclear reactor and detection of concealed fissionable materials are among the applications of these detectors. Fast neutrons can be detected as a result of nuclear reactions that produce charged-particle products. One of promising material is Silicon Carbide(SiC) which has wide band-gap energy from 2.2 to $3.2 \mathrm{eV}$ for the most common poly-types. A large energy band gap has the possibility of low leakage currents, a good radiation resistance and a reasonable sensing capability for the charges created during an ionization process. ${ }^{4-6)}$ Commercial

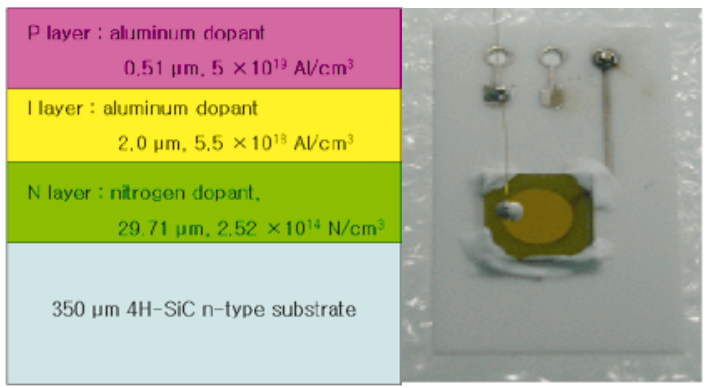

Fig. 1 Detector Schematics and PIN Neutron Detector

*Corresponding Author, E-mail:jhha@kaeri.re.kr

(C) Atomic Energy Society of Japan available single crystals of $\mathrm{SiC}$ are the hexagonal $4 \mathrm{H}-\mathrm{SiC}$ and $6 \mathrm{H}-\mathrm{SiC} .4 \mathrm{H}-\mathrm{SiC}$ single crystal wafer was used for fabrication and characterization of neutron detector

The present work focuses on the development of a fast neutron semiconductor detector based on a wide band-gap $\mathrm{SiC}$ semiconductor, operating at a zero biased voltage by using a strong internal electric field due to a PIN structure.

\section{Experimental Procedures}

\section{4H-SiC PIN-type Detector Fabrication}

We fabricated a PIN type of $\mathrm{SiC}$ radiation detector. PIN structured wafer was provided by Cree Co. (Item No. DG0798-04), which consisted of a 4 layer structure with $0.5 \mu \mathrm{m}\left(5 \times 10^{19} / \mathrm{cm}^{3}\right)$ of a P-layer, $2 \mu \mathrm{m}\left(5.5 \times 10^{18} / \mathrm{cm}^{3}\right)$ of a $\mathrm{N}$-layer and $30 \mu \mathrm{m}\left(2.52 \times 10^{14} / \mathrm{cm}^{3}\right)$ of a highly doped N-layer on a $350 \mu \mathrm{m}$ N-type substrate. Electrode metals, $\mathrm{Ni}$ and $\mathrm{Au}$, were deposited on the $5 \times 5 \mathrm{~mm}^{2}$ wafer substrate with $3 \mathrm{~mm}$ in diameter to extract the charge signals. The electrodes of $\mathrm{SiC}$ consisted of $\mathrm{Ni}(30 \mathrm{~nm}) / \mathrm{Au}(100 \mathrm{~nm})$ on Si-face and $\mathrm{Ni}(30$ $\mathrm{nm}) / \mathrm{Au}(200 \mathrm{~nm})$ on the $\mathrm{C}$-face. The thin Au surface designed to penetrate sufficiently by several $\mathrm{MeV}$ charged particles which used for charge collection characterization.

\section{I-V Characteristics Measurement}

To measure the current (I)-voltage (V) characteristics, the detector mounted on a ceramic substrate with a $1 \mathrm{~mm}$ diameter Mo-Mn electrode. A SiC detector was fixed with a high temperature conducting epoxy onto the ceramic layer and connected by a gold wire to extract the electric signal. 
I-V characteristics of detector were measured by using the HP 4155B, a semiconductor parameter analyzer with a self voltage sources by adjusting the internal bias voltage within $-40 \mathrm{~V}$ to $10 \mathrm{~V}$ in $0.1 \mathrm{~V}$ intervals. I-V measurements were carried out at room temperature in a box shielded from the light. The SiC detector with preamplifier was mounted in the shielding box to protect external noise sources and light.

\section{Neutron Response Measurement}

Neutron Detection response was measured by using an Am-Be neutron source with $5.0 \mathrm{MeV}$ in average energy. Neutron flux on the detectors was a $1.1 \times 10^{6} \mathrm{n} / \mathrm{cm}^{2} / \mathrm{sec}$. Fast neutrons can be detected by measuring secondary charge paticles which produced by the incident neutrons. While neutrons are incident into semiconductor, neutron reacted with $\mathrm{Si}$ and $\mathrm{C}$ nuclei in $\mathrm{SiC}$ semiconductor, and then $\mathrm{Si}$ and $\mathrm{C}$ ions are recoiled. The recoiled energetic ions are absorbed in the $\mathrm{SiC}$ depleted sensor region, and generate electrons and holes which number of pair is proportional to the incident neutron energy. By using internal electric field, these electrons are collected by electrode and extracted by readout pad to produce a current pulse.

The $\mathrm{SiC}$ detector and the preamplifier based on hybrid chip CR110 which is provided by Cremat Co. was mounted in a shielding box to protect noise and light. Neutron spectra were obtained by feeding preamplifier signals into ORTEC's shaping amplifier, a multi-channel analyzer (MCA), and DAQ system.

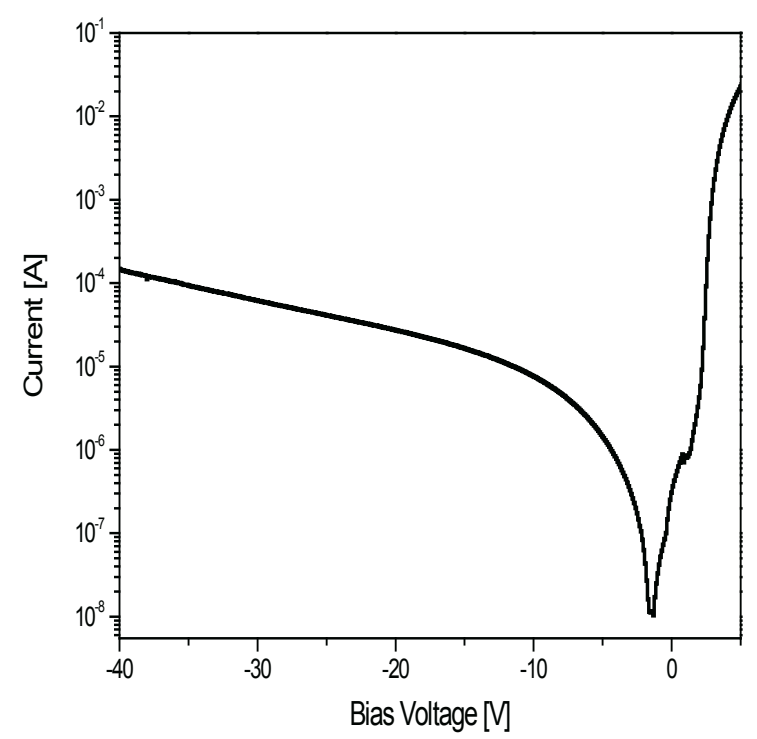

Fig. 2 The typical current-voltage characteristics measurement result. Forward current upper limit was set as $50 \mathrm{~mA}$ as seen at forward current region.

\section{Results and Discussion}

\section{I-V Characteristics Measurement}

Current-voltage measurement with respect to a biased voltage is a major electrical property in detector. Figure $\mathbf{2}$ is the typical I-V characteristics. While measuring I-V curve upper current limit was set as $50 \mathrm{~mA}$ for protection from the forward big current damage. Exponential behavior for forward bias region is explained as the minority carrier diffusion currents of electron or hole. PIN structure device compensated by boron dopant showed similar I-V pattern. ${ }^{7 \text { ) }}$

\section{The Charge Collection Efficiency (CCE) Measurement}

The alpha-ray response by using $5.5 \mathrm{MeV}$ energy of alpha-rays from ${ }^{241} \mathrm{Am}$ source was used to determine CCE. Pulse height spectra were obtained by using NIM standard electronics as a function of biased voltages. This is also reconfirmed by extracting the charge collection CCE curve as the function of a biased voltage up to $25 \mathrm{~V}$ as shown in Fig. 3. As the biased voltage increases, the $5.5 \mathrm{MeV}$ alpha-ray peak channel number increase and finally peak channel number saturated when reached at $100 \%$ of CCE. Over $25 \mathrm{~V}$ of a biased voltage the alpha-ray peak positions almost overlapped in pulse height spectra, because the charges are fully collected in the detector. Even though at zero biased voltage, the CCE reached about $65 \%$ by the internal built-in voltage (potential) which originated from the electric field inside the PIN detector structure.

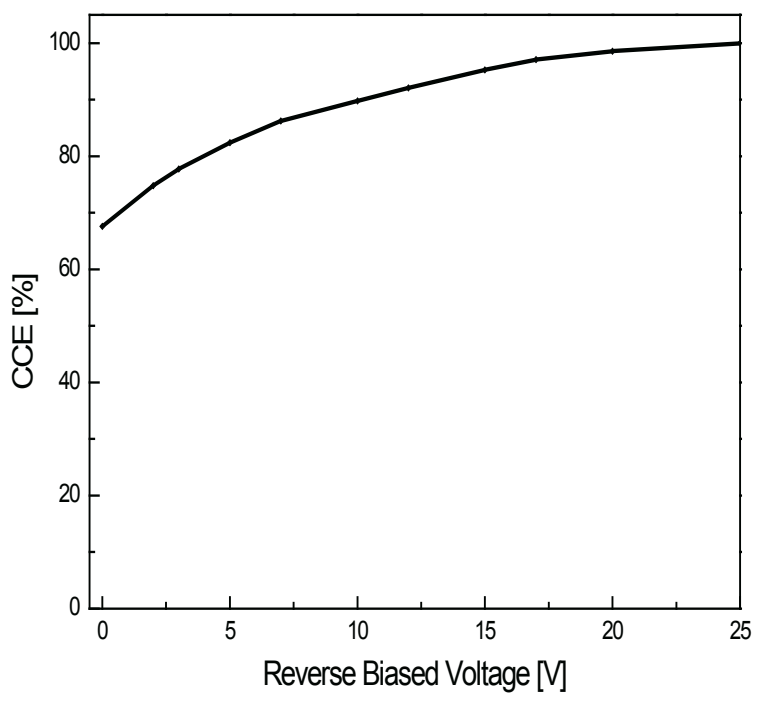

Fig. 3 The Charge collection efficiency (CCE) as a function of the reverse biased voltage. 
The built-in voltage can be calculated by ${ }^{9)}$

$$
\mathrm{V}_{\mathrm{bi}}=\frac{\mathrm{kT}}{\mathrm{q}} \ln \left(\frac{\mathrm{N}_{\mathrm{A}} \mathrm{N}_{\mathrm{D}}}{\mathrm{N}_{\mathrm{i}}^{2}}\right)
$$

Where $\mathrm{N}_{\mathrm{A}}$ is acceptor concentration $\left(5.5 \times 10^{18} / \mathrm{cm}^{3}\right), \mathrm{N}_{\mathrm{D}}$ is doner concentration $\left(2.52 \times 10^{14} / \mathrm{cm}^{3}\right)$, and $\mathrm{N}_{\mathrm{i}}$ is intrinsic carrier density. Where $\mathrm{N}_{\mathrm{i}}$ was assumed as $1.0 \times 10^{-8} / \mathrm{cm}^{3}, \mathrm{~V}_{\mathrm{bi}}$ was extracted as $2.93 \mathrm{~V}$. Depletion depth and maximum internal electric field can be obtained by a reference [8]. The depletion depth at zero biased voltage was obtained as a 3.6 $\mu \mathrm{m}$. The maximum internal electric field strength was a 16.3 $\mathrm{kV} / \mathrm{cm}$, where the dielectric constant of $4 \mathrm{H}-\mathrm{SiC}$ was used for as a 10.03. Such strong internal electric field gives a possibility to operate without external power supply and other extra long cable in detection system.

\section{Neutron Response Measurement}

Neutron detection responses were measured up to a $1.1 \times 10^{6}$ $\mathrm{n} / \mathrm{sec}-\mathrm{cm}^{2}$ at a self-powered operation mode. The main reactions of the neutron detection response are interpreted as the ${ }^{12} \mathrm{C}\left(\mathrm{n}, \mathrm{n}^{\prime}\right){ }^{12} \mathrm{C}$ and ${ }^{28} \mathrm{Si}\left(\mathrm{n}, \mathrm{n}^{\prime}\right){ }^{28} \mathrm{Si}$ elastic and inelastic scattering. The carbon-recoil ion continuum can be seen to extend to higher energies as shown in Fig. 4, because more neutron kinetic energy can be transferred to the recoiled carbon ion than to the recoiled silicon ion. The relatively thick diode structures are sensitive to the gamma-ray response originated from $\mathrm{Am}-\mathrm{Be}$ reaction, which contributes low energy tail. Below 40 of the channel number in Fig.4 is considered due to the gamma-ray and background contribution. Background was measured before experiment. Neutron contribution was selected as the events obtained up to 40 of channel number in spectrum.

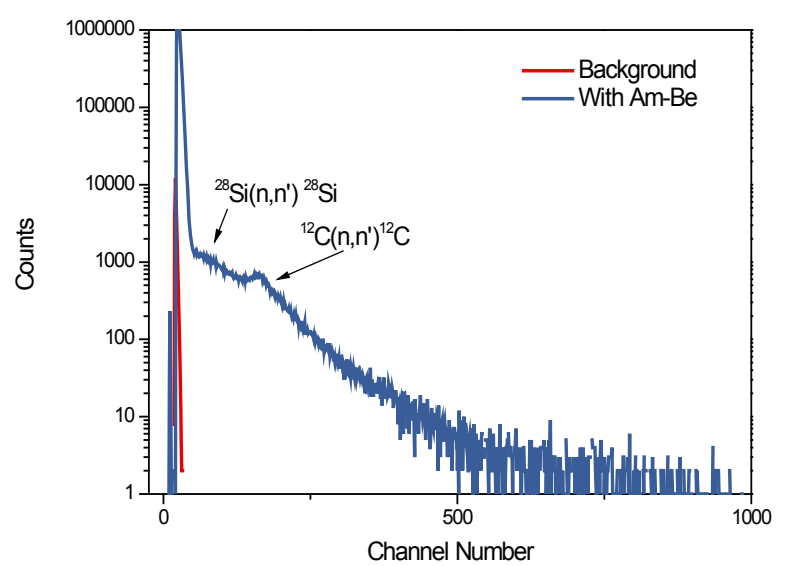

Fig. 4. Fast neutron spectrum for $5.0 \mathrm{MeV}$ neutron energy. Detector was operating without a biased voltage (zero-=biased mode)

The distance between the detector and neutron source was a $1.2 \mathrm{~cm}$ and the active area of detector was a $0.07065 \mathrm{~cm}^{2}$.
Also, the neutron response of detector was measured as a $39.9 \mathrm{n} / \mathrm{sec}$. By considering active area the absolute neutron detection efficiency was determined as a $5.1 \times 10^{-4}$ for a 5.0 $\mathrm{MeV}$ of fast neutrons.

\section{Conclusion}

The 4H PIN SiC radiation detector was fabricated with 3 layer-structures which were $0.5 \mu \mathrm{m}$ of a P-layer, $2 \mu \mathrm{m}$ of a I-layer and $30 \mu \mathrm{m}$ of a N-layer on a $350 \mu \mathrm{m}$ N-type substrate. The detector was designed and fabricated to operate at zero bias voltage for neutron detection at room temperature. Electrical and radiation response properties of detector were measured such as current-voltage characteristics, alpha and neutron response. The fabricated PIN SiC detectors performance showed a possibility as a neutron detector for harsh environment by operating at a self-powered mode.

\section{Acknowledgment}

This work has been carried out under the nuclear R\&D program of the Ministry of Education, Science and Technology (MEST).

\section{References}

1) M. Rahman et. al., "Super-radiation hard detector Technologies: 3D- and widegap detectors", IEEE. Trans. on Nucl. Sci. vol. 51 no. 5, 2256 ( 2004)

2) A. R. Dulloo, et. al, "The thermal neutron response of miniature silicon carbide semiconductor detectors", Nucl. Inst. Meth A 498,. 415 - 423, 2003

3) F. H. Ruddy, et. al., "The fast neutron response of $4 \mathrm{H}$ silicon carbide semiconductor radiation detectors', IEEE Trans. On Electron Devices, vol. 53, no. 3, 1666 (2006)

4) S. Seshadri, et. al., "Demonstration of an $\mathrm{SiC}$ Neutron Detector for High-Radiation Environments", IEEE Trans. On Electron Devices, vol. 46, no. 3, 567 (1999)

5) J. H. Ha, Y. K. Kim, "Fabrication and Characteristics of a Fully Depleted Semi-Insulating GaAs Semiconductor Detector for Alpha Radiation Detection at Room Temperature in Air", $J$. Korean Phys. Soc. vol. 52, pp. 576 - 579, 2008

6) M. Rogalla, K. Runge, A. Soldner-Rembold, "Paticle detectors based on semi-insulating Silicon Carbide", Nucl. Phys. B(Proceedings Supplements) vol. 78, pp. 516-520, 1999

7) M. Badila et. al., "Current-voltage characteristics of large area 6H-SiC pin diodes”, Mat. Sci. and Eng. B61-62, 433 (1999)

8) G. Lutz, "Semiconductor radiation detectors" , Springer-Verlag, ch.3-5 (1999) 